FeNO and pulmonary functions. Three studies were not significant correlation. The various results of studies were effected by characteristics of the patients (COPD severity, smoking status, treatment status) and differences in FeNO measurement methods. Conclusions The studies includes in this review highlight the difficulties of correlation between FeNO and pulmonary function. So, the role of add-on monitoring of FeNO to pulmonary function test is less clear because of the absence of conclusive double-blind, randomised, control studies concerning potential clinical benefits in the management of COPD. Further randomised controlled trials are required.

\section{PARENTAL INCOME IS MORE IMPORTANT THAN PARENTAL EDUCATION TO CHILDREN'S HEALTH AND WELLBEING IN ADULTHOOD: EVIDENCE FROM THE TROMSØ STUDY}

Mashhood Ahmed Sheikh. Department of Community Medicine, University of Tromso, Tromso, Troms, Norway

\subsection{6/oemed-2014-102362.374}

Objectives The aim of this research was to estimate and compare the direct and indirect influence (mediated by respondents' education) of three indicators of CSES (childhood financial conditions, mothers' education, fathers' education) on: i) the generic health dimensions included in the EQ-5D; ii) self-rated health $(\mathrm{SRH})$, iii) age-comparative self-rated health (ASRH), and; iv) subjective wellbeing.

Method The data was analysed using Stata command Paramed. Log-linear regression was used for the health and life satisfaction outcomes to estimate the natural direct effects (NDE), natural indirect effects (NIE) and marginal total effects (MTE) as risk ratios (RR). Statistically significant interaction $(p<0.05)$ was observed between the CSES exposures and gender, regressed on the health and wellbeing outcomes, therefore the analysis was conducted separately for men and women.

Results Childhood financial conditions was associated (NDE) with all health measures. Men had a higher risk of being unhealthy on the composite EQ-5D measure, and the anxiety/depression dimension, but women had a higher risk of being unhealthy on the dimensions self-care, usual activities, pain/discomfort, as well as on SRH. Childhood financial conditions had no statistically $(p>0.05)$ significant NIE mediated by respondents' education, on any health measure. While almost all NDEs of parental education on health outcomes were not statistically significant ( $p$ $>0.05)$, most of the NIEs of parental education were statistically significant $(\mathrm{p}<0.05)$.

Conclusions Childhood financial conditions have a strong direct effect on later health and wellbeing, independent of respondents' education, while parental education has an indirect effect on later health mediated by respondents' education.

\section{Symposiums}

\section{COMPONENTS OF THE HEALTHY WORKER EFFECT WITH QUANTIFICATION FOR DIFFERENT REFERENT COMPARISONS}

J Morel Symons, Kim Kreckmann, Hien Le, Sarah Starks. DuPont Epidemiology Program, Newark, DE, USA

10.1136/oemed-2014-102362.375
Objectives The healthy worker effect (HWE) is widely known to bias standardised risk estimates from occupational cohort studies. Multiple factors contribute to HWE bias that is commonly characterised as confounding due to the selection of individuals with "better health status" who are more likely to gain and retain employment relative to a general population including non-employed persons. Comparisons between standardised mortality ratios (SMRs) estimated from reference population rates with different characteristics allow for quantitative evaluation of different components of the HWE.

Method Data from over five decades for a company-wide mortality registry comes from life insurance claims, and deaths are validated against the U. S. National Death Index. Average personyears at risk during five-year calendar periods for the occupational cohort population are estimated. The expected mortality counts are specific to age, sex, race, and calendar-time period strata. SMRs are calculated based on the mortality rates for the general U.S. population and the company-wide population.

Results From 1956 through 2012, the annual US employee population has ranged from 29000 to 108000 workers. The mortality registry includes over 80000 deaths validated through 2010, 25\% due to malignant neoplasms and 37\% due to cardiovascular diseases.

Conclusions The HWE influences the interpretation of standardised estimates from occupational studies. Comparisons for different reference populations can evaluate differential HWE bias of associations between occupational exposure and mortality. Analyses based on company reference rates identify contributions from components of the HWE based on comparable demographic characteristics, a similar likelihood of obtaining and retaining employment, and an equivalent potential for ascertainment of mortality outcomes.

\section{A BAYESIAN APPROACH TO ACCOUNT FOR THE HEALTHY WORKER SELECTION EFFECT}

${ }^{1}$ Igor Burstyn, ${ }^{2}$ Ghassan Harma, ${ }^{3}$ J Morel Symons. 'Drexel University, Philadelphia, PA, USA; ${ }^{2}$ International Agency for Research on Cancer, Lyon, France; ${ }^{3}$ DuPont, Willmington, $D E, U S A$

\subsection{6/oemed-2014-102362.376}

Objectives We propose a Bayesian method to adjust for the component of the healthy worker effect that arises from selection of healthier individuals into workforce to allow correct estimation of the standardised mortality ratio (SMR) and associated credible intervals.

Method Information on general populations is typically used to generate expected counts for outcomes in SMR calculations but an occupational cohort is not a random sample of the general population. The alternative is to use the expected number of outcomes from industrial cohorts known to experience the outcome of interest but free of the exposures that defined the observed cohort. In Bayesian terms, we can view "expected counts of outcomes given the observed age-sex-period structure" as the target of inference for which we seek a posterior distribution. We show that the problem reduces to elucidation of a prior distribution: we propose using expert opinions about relative rates of mortality outcomes of interest in the observed cohort relative to general population rates and direct estimation of reference rates from occupational cohort studies.

Results Data from DuPont on $320000+$ active and former employees with work histories in the US from 1955 will be used. This registry allows for the calculation of expected 